\title{
Abscesos cerebrales: análisis de factores pronóstico e influencia del tratamiento antibiótico protocolizado en su evolución
}

\author{
J. GÓMEZ, E. GARCÍA-VÁZQUEZ, M. MARTÍNEZ PÉREZ, J. MARTÍNEZ LAGE', \\ J. GONZÁLEZ TORTOSA' ${ }^{1}$, M. A. PÉREZ ESPEJO ${ }^{1}$, J. RUIZ' ${ }^{1}$, J. A. HERRERO ${ }^{2}$, \\ M. CANTERAS ${ }^{3} \cdot M$. VALDÉS ${ }^{4}$
}

Servicio Medicina Interna-Infecciosas. ${ }^{\text {SServicio de Neurocirugía } . ~}{ }^{2}$ Servicio de Microbiología. ${ }^{3}$ Departamento de Bioestadística. Hospital Universitario Virgen de la Arrixaca. Departamento de Medicina Interna. Facultad de Medicina. Universidad de Murcia

\begin{abstract}
BRAIN ABSCESS. EVALUATION OF PROGNOSTIC FACTORS: DOES THE USE OF ANTIBIOTIC PRESCRIBING PROTOCOLS IMPROVE OUTCOME?
\end{abstract}

\section{RESUMEN}

Objetivos: Analizamos los factores pronósticos asociados a mortalidad en pacientes con absceso cerebral (AC) y la influencia del tratamiento antibiótico protocolizado (PA).

Pacientes y Métodos: estudio observacional en un hospital universitario (1975-2005). El tratamiento antibiótico empírico se realizó sin PA (Periodo I -PI-, 1975-1983; n = 33) y de acuerdo a PA diseñado por los Servicios de MI-Infecciosas y Neurocirugía a partir de 1983 (Periodo II PII-; $\mathrm{n}=71$ ); valoramos la influencia del Protocolo Terapéutico en la curación de los enfermos.

Resultados: se evaluaron 104 pacientes (edad media 45 años; rango 12-86); el origen del AC se identificó en $89 \%$ y en $76 \%$ hubo confirmación microbiológica. Mortalidad, 16,3\% (17/104); se asociaron de forma estadísticamente significativa a mayor mortalidad: edad $>40$ años, enfermedad de base últimamente fatal, situación clínica inicial crítica-mala, presencia de alteración del estado de conciencia y tratamiento empírico no adecuado. No hubo diferencias estadísticamente significativas en las características epidemiológicas, clínicas, radiológicas o microbiológicas de los pacientes correspondientes al PI y al PII, excepto en edad $(>40$ años, $36 \%$ en PI y $62 \%$ en PII); si hubo diferencias en curaciones, $60 \%$ vs $77 \%(\mathrm{p}<0,05)$; recidivas 21 vs. $7 \%(\mathrm{p}<0,05)$ y mortalidad $18 v s$. $15,4 \%(\mathrm{p}>0,05)$ en PI y PII, respectivamente.

Conclusiones: La edad, enfermedad de base últimamente fatal, gravedad clínica inicial, presencia de alteración del nivel de conciencia y el tratamiento antibiótico empírico inadecuado son los principales factores pronósticos asociados a mayor mortalidad en pacientes con AC. La prescripción del tratamiento empírico de acuerdo con un PA consensuado se asoció con mayor porcentaje de curaciones y menor número de recidivas.

PALABRAS CLAVE: Absceso cerebral. Factores pronósticos. Tratamiento empírico. Protocolo antibiótico.

\section{ABSTRACT}

Background: the aim of this study was to evaluate prognostic factors in brain abscess $(A B)$ and influence of management with antibiotic prescribing protocols $(A P P)$.

Patients and Methods: observational study of a cohort of non-paediatric patients with BA admitted at a 944-bed hospital (1976-2005). Data collection from clinical records has been done according to a standard protocol. We analysed epidemiological, clinical, radiological, microbiological and laboratory data associated with mortality. From 1976 to 1983 (Period I), antibiotic treatment was not done according to any internal APP; from 1983 (Period II), antibiotic management was done according to a APP designed by infectious diseases specialists and neurosurgeons. Predictors of mortality were identified by univariate analysis. The influence of the use of APP in outcome was assessed.

Results: 104 patients with BA were included (mean age 45 years, range 12-86); presumed primary pathogenic mechanism of $B A$ was identified in 89\%; microbiologic diagnosis was made in $76 \%$. Overall mortality was $16.3 \%$. Factors statistically associated with higher mortality were: age $>40$ years, ultimately fatal underlying disease, acute severe clinical condition at the onset of BA, altered mental status and inadequate empirical treatment; 33 patients were treated in Period I and 71 in Period II; no statistically significant differences were found between epidemiological, clinical, radiological or microbiological characteristics of the groups except for mean age (> 40 years in $36 \%$ and $62 \%$ respectively in Period I and II). Rates of resolution of BA were $60 \mathrm{vs} .77 .4 \%(p<$ $0.05)$; relapses 21 vs. $7 \%(p<0.05)$ and mortality $18 v s .15 .4 \%(p>$ $0.05)$, in Period I and II respectively.

Conclusions: main prognostic factors associated with mortality in patients with $B A$ are age, rapidly fatal underlying disease, acute severe clinical condition at the onset of BA, altered mental status and inadequate empirical treatment. Empiric treatment according to APP was associated with greater resolution and lower relapse rates.

KEY WORDS: Brain access. Prognostic factors. Empirical treatment. Antibiotic prescribing protocols.

Gómez J, García-Vázquez E, Martínez Pérez M, Martínez Lage J, Gónzalez Tortosa J, Pérez Espejo MA, Ruiz J, Herrero JA, Canteras M. Abscesos cerebrales: análisis de factores pronóstico e influencia del tratamiento antibiótico protocolizado en su evolución. An Med Interna (Madrid) 2008; 25: 331-334.

\section{INTRODUCCIÓN}

Los abscesos cerebrales son unos procesos supurativos localizados en el parénquima cerebral relativamente infre- cuentes pero de gran relevancia clínica debido a su elevada morbi-mortalidad. La sintomatología clínica inicial puede ser poco específica de infección, lo que dificulta la sospecha diagnóstica precoz y condiciona una elevada mortalidad (1),

Trabajo aceptado: 14 de marzo de 2008 
que puede llegar a ser de hasta $30-40 \%$ y que a pesar de los avances diagnósticos y terapéuticos no se ha modificado en los últimos años (2). Entre los factores que tradicionalmente se han asociado con un peor pronóstico destacan el grado de deterioro del nivel de conciencia en el momento del diagnóstico, la presencia de abscesos múltiples, la presentación aguda del cuadro, la edad avanzada y el tratamiento antibiótico inadecuado. La pretensión de este trabajo es analizar nuestra experiencia de 30 años, valorando los factores pronósticos asociados a mayor mortalidad en estos pacientes, así como evaluar la influencia que el uso protocolizado del tratamiento antibiótico consensuado tiene en su evolución.

\section{PACIENTES Y MÉTODOS}

Se han estudiado de forma consecutiva, desde octubre de 1976 hasta final de 2005, 108 pacientes diagnosticados de absceso cerebral (AC) e ingresados en el Hospital Universitario Virgen de la Arrixaca de Murcia, único hospital con Servicio de Neurocirugía en la Comunidad Autónoma de Murcia, la cual cuenta con 1 millón doscientos mil habitantes. Parte de esta serie ya fue analizada en otro trabajo de nuestro grupo (3).

El diagnóstico de AC se sospechó por la clínica y criterios radiológicos (TAC o angiografía), confirmándose por cirugía y anatomía patológica y/o microbiología en 104 casos. Se han excluido 4 casos: dos pacientes que fallecieron en las primeras 48 horas y en los que el diagnóstico se hizo en la necropsia y otros dos en los que la clínica y el TAC eran compatibles con $\mathrm{AC}$, pero en los que no pudo confirmarse el diagnóstico por presentar insuficiencia respiratoria grave que contraindicó la neurocirugía haciéndose tratamiento antibiótico empírico.

\section{TIPO DE PACIENTES}

A todos los pacientes se les realizó historia clínica y exploración física completa, valorándose especialmente los antecedentes de infecciones previas, los síntomas que motivaron la consulta clínica y la exploración neurológica al ingreso.

Los pacientes fueron clasificados según: a) pronóstico de la enfermedad de base de acuerdo con los criterios de McCabe y Jackson (4), (I, rápidamente fatal; II, últimamente fatal y III, no fatal); b) estado de conciencia (A, alerta; B, confusión y obnubilación; $\mathrm{C}$, respuesta solo a estímulos dolorosos; D, ausencia de respuesta a estímulos o "coma profundo"); y c) situación de gravedad clínica inicial siguiendo los criterios de Winston et al. (5) (crítica, mala, regular y estable). Diariamente y durante toda la hospitalización se realizaron sucesivos controles clínicos. Posteriormente tras el alta hospitalaria, se realizó seguimiento de todos los pacientes por consulta externa cada 3-4 meses hasta completar un año.

\section{ESTUDIOS COMPLEMENTARIOS}

De forma rutinaria se realizaron estudios hematológicos, de coagulación y bioquímicos, que se repitieron durante el curso clínico y al final del mismo.

Todos los pacientes se sometieron a estudio con TAC el primer día de sospecha clínica, repitiéndose, si no había complicaciones, cada dos o tres semanas y después cada cuatro meses hasta completar el año de seguimiento.

\section{ESTUDIOS MICROBIOLÓGICOS}

En todos los casos confirmados (104), se recogieron muestras apropiadas del lecho del absceso, que se procesaron para cultivo en medios aerobios que incluían (agar-sangre, agar-chocolate, tioglicolato y McConkey) y en medios anaerobios (en cámara de anaerobios o Gaspaks), utilizando como medios agar-sangre, Schaedler KV y agar-gentamicina. La identificación de los microrganismos aislados se realizó de acuerdo con los criterios aceptados a nivel internacional. La susceptibilidades frente a los distintos antimicrobianos se valoraron por el método disco-placa siguiendo los criterios de la CLSI (Clinical and Laboratory Standards Institute) (6). El estudio microbiológico se repitió en caso de que por cualquier circunstancia el paciente hubiera tenido que ser intervenido quirúrgicamente de nuevo en el curso del tratamiento.

\section{TRATAMIENTO ANTIBIÓTICO}

El periodo de estudio se dividió en dos fases: Periodo I (PI) en el que se evaluaron 33 pacientes que recibieron tratamiento antibiótico de acuerdo con el criterio del médico responsable, sin atenerse a protocolo consensuado (1976-1983) y Periodo II, en el que se incluyen 71 pacientes que recibieron tratamiento antibiótico de acuerdo a un protocolo diseñado por el Servicio de MI-Infecciosas en conjunción con el Servicio de Neurocirugía.

En el PI, los pacientes se trataron con las siguientes combinaciones: penicilina y cloranfenicol $(n=23)$, ampicilina y gentamicina $(n=4)$, cefazolina y gentamicina $(n=3)$ y carbenicilina y amikacina $(n=1)$. Los pacientes del PII recibieron como tratamiento empírico la combinación de cefotaxima y metronidazol y ceftacidima o meropenem junto con cloxacilina o vancomicina en caso de antecedente neuroquirúrgico o traumatismo craneo-encefálico (TCE), ajustándose posteriormente de acuerdo con los resultados microbiológicos y/o evolución clínica. El tratamiento se inició en las primeras 24 horas tras la confirmación diagnóstica quirúrgica. La duración del tratamiento osciló entre 21 y 42 días. De forma complementaria los pacientes recibieron dexametasona a dosis de 8 mg cada 8-12 horas durante la fase aguda y antiepilépticos de forma empírica.

Se consideró que el tratamiento antibiótico era adecuado cuando los microrganismos aislados eran sensibles al menos a uno de los antimicrobianos utilizados o, en caso de no disponer de documentación microbiológica, cuando se producía la mejoría clínica y radiológica del paciente.

\section{CRITERIOS DE VALORACIÓN DE LA EFICACIA TERAPÉUTICA}

Para valorar la eficacia de las medidas terapéutica empleadas se tuvieron en cuenta tres parámetros: curación, recidiva y fallecimiento. Se consideró curación, cuando se produjo la desaparición de la clínica y la mejoría de las imágenes radiológicas en la TAC; recidiva, cuando reaparecía la clínica tras la mejoría inicial, con empeoramiento de las imágenes de la TAC y necesidad de nuevo drenaje quirúrgico; y fallecimiento cuando el paciente moría antes de la resolución del AC y en ausencia de otras causas que lo justificasen (7). 


\section{ANÁLISIS ESTADÍSTICO}

Los factores pronósticos de todos los pacientes y las características de base de ambos grupos terapéuticos se sometieron a un estudio de tablas de contingencia variables, mediante el test de Pearson, con objeto de determinar aquellas variables asociadas de forma estadísticamente significativa a mayor mortalidad o recidiva. Se compararon los pacientes del PI y PII para detectar diferencias significativas entre ambos.

\section{RESULTADOS}

La incidencia de abscesos cerebrales en nuestro hospital en el periodo 1976-1989 fue de 4-5 casos por millón de habitantes/año y entre 1990-2005 ha pasado a ser de 2-3 casos por millón de habitantes/año.

Los 104 pacientes que tenían confirmación diagnóstica tenían una edad media de 45 años (rango 12-86), de los cuales 64 eran hombres y 40 mujeres.

No hubo diferencias estadísticamente significativas en las características epidemiológicas, clínicas, radiológicas o microbiológicas de los pacientes correspondientes al Periodo I (PI) y al Periodo II (PII), excepto en cuanto a la edad ( $>40$ años, $36 \%$ en PI y $62 \%$ en PII; p < 0,05) (Tabla I).

La mortalidad fue del 16,3\% (17/104); se asociaron de forma estadísticamente significativa a mayor mortalidad: edad $>$ 40 años, enfermedad de base últimamente fatal, situación clínica inicial crítica-mala, presencia de alteración del estado de conciencia y el tratamiento empírico no adecuado.

El porcentaje de curaciones fue del 60 vs. 77,4\% (p < $0,05)$; recidivas $21 v s .7 \%$ ( $<<0,05)$ y mortalidad $18 v s$. $15,4 \%(\mathrm{p}>0,05)$ en los pacientes correspondientes al PI y PII, respectivamente.

\section{DISCUSIÓN}

Los abscesos cerebrales constituyen uno de los procesos con mayor morbi-mortalidad en las unidades neuroquirúrgicas, tradicionalmente asociada a la gravedad clínica inicial, la presencia de enfermedad de base crónica y especialmente al tratamiento antibiótico empírico inadecuado $(3,8)$.

La mortalidad de nuestra serie $(16,3 \%)$, se encuentra dentro de los márgenes reseñados en la literatura reciente $(9,10)$ sobre abscesos cerebrales, que oscila entre 13 y $22 \%$, siendo menor que las observadas en otros centros, como el Kennyata Nacional Hospital (11) (30,7\%) y el Bichat Claude Berbard Hospital (12) (26\%), cuyas cifras están probablemente relacionadas con la mayor presencia de pacientes con infección por el VIH en el caso del primero y con el ingreso en UCI de los pacientes en coma profundo en el segundo, y discretamente superior a la descrita por Carpenter et al., (13) en una serie de 49 pacientes con abscesos cerebrales, cuya menor mortalidad $(10 \%)$ podría explicarse por el predominio en su serie de AC postraumáticos en pacientes jóvenes. El porcentaje de enfermos con documentación microbiológica de nuestra serie (76\%) es semejante al de otros estudios $(8,10,11)$.

A pesar de todos los avances en el diagnóstico de estas infecciones (TAC, RMN), la morbilidad y mortalidad asociadas a los AC permanecen sin cambios significativos $(3,8)$. Se hace necesario por tanto reflexionar sobre cuales son los fac-
TABLA I

CARACTERÍSTICAS DE LOS PACIENTES SEGÚN PERIODO DE TRATAMIENTO ANTIBIÓTICO

\begin{tabular}{|c|c|c|c|}
\hline Características & $\begin{array}{c}\text { Periodo I } \\
(n=33)(\%)\end{array}$ & $\begin{array}{l}\text { Periodo II } \\
(n=71)(\%)\end{array}$ & $p$ \\
\hline Edad > 40 años & $12(36)$ & $44(62)$ & $<0,05$ \\
\hline \multicolumn{4}{|l|}{ Sexo } \\
\hline Hombre & $19(57,6)$ & $45(63,4)$ & \multirow[t]{2}{*}{ ns } \\
\hline Mujer & $14(42,4)$ & $26(36,6)$ & \\
\hline \multicolumn{4}{|l|}{ Enfermedad de base (McCabe) } \\
\hline I & 0 & 0 & \\
\hline$\|$ & $13(39,4)$ & $19(26,8)$ & \multirow[t]{2}{*}{ ns } \\
\hline III & $20(60,6)$ & $52(73,2)$ & \\
\hline \multicolumn{4}{|l|}{ Tipo de Coma } \\
\hline A & $15(45,5)$ & $34(47,9)$ & \multirow[t]{2}{*}{ ns } \\
\hline$B, C O D$ & $18(54,5)$ & $37(52,1)$ & \\
\hline \multicolumn{4}{|c|}{ Gravedad Clínica Inicial (Winstons et al) } \\
\hline Crítica & $4(12,1)$ & $6(8,4)$ & \multirow{3}{*}{ ns } \\
\hline Mala & $15(45,5)$ & $36(50,7)$ & \\
\hline Regular-Estable & $14(42,4)$ & $29(40,8)$ & \\
\hline \multicolumn{4}{|l|}{ Factores Predisponentes } \\
\hline Infecciones de otra localización & $29(87,9)$ & $61(85,6)$ & \multirow{4}{*}{ ns } \\
\hline Cirugía Craneal previa & $12(36,4)$ & $36(50,7)$ & \\
\hline TCE & $5(15,1)$ & $14(19,7)$ & \\
\hline Otros & $6(18,2)$ & $5(7)$ & \\
\hline \multicolumn{4}{|l|}{ Documentación Microbiológica } \\
\hline Cocos Grampositivos & $22(66,7)$ & $58(81,7)$ & \multirow{4}{*}{ ns } \\
\hline Bacilos Gramnegativos & $12(36,4)$ & $33(46,5)$ & \\
\hline Anaerobios & $8(24,2)$ & $12(16,9)$ & \\
\hline Otros & $2(6)$ & $13(18,3)$ & \\
\hline \multicolumn{4}{|l|}{ Localización } \\
\hline Temporal & $14(42,4)$ & $19(26,8)$ & \multirow{5}{*}{ ns } \\
\hline Parietal & $7(21,2)$ & $15(21)$ & \\
\hline Frontal & $7(21,2)$ & $25(35,2)$ & \\
\hline Cerebeloso & $2(6)$ & $8(11,3)$ & \\
\hline Otros & $3(9)$ & $10(14)$ & \\
\hline
\end{tabular}

tores pronósticos más importantes y cuales podemos modificar para conseguir una mejor supervivencia y disminución de secuelas en estos pacientes. En este sentido, tenemos que señalar que en nuestra serie y al igual que en la serie inicial (8), el principal factor pronóstico asociado a mayor mortalidad es el tratamiento antibiótico empírico inadecuado, además de la gravedad clínica inicial de los pacientes y la alteración del estado de conciencia al diagnóstico, datos que coinciden con los de otras series de la literatura (8-11). Como en otros procesos infecciosos graves, comprobamos como la presencia de enfermedad de base del grupo últimamente fatal (criterios de McCabe et al.), figura de forma significativa como factor asociado a peor pronóstico. Si consideramos que de todos estos factores solo podemos actuar sobre la elección del tratamiento antibiótico empírico, haciendo que sea lo más óptimo posible y por tanto adecuado, quedan sentadas las bases que justifican la necesidad de la implantación de un Protocolo Terapéutico consensuado con los Servicios de Neurocirugía, el cuál debe tener en cuenta las características clínico-epidemiológicas de los pacientes con AC, así como los datos 
microbiológicos propios del hospital (en lo que hace referencia a AC post-quirúrgicos). En nuestro centro y a partir de 1983 se implantó un protocolo de tratamiento antibiótico empírico de acuerdo con las Guías Clínicas que recomendaba tratamiento combinado de cefotaxima y metronidazol, en base a la gran actividad antimicrobiana del primero frente a microrganismos grampositivos y bacilos gramnegativos más frecuentemente aislados en estos procesos cuando no se relacionan con antecedente neuroquirúrgico o TCE (Streptococcus del grupo viridans y enterobacterias $-P$. mirabilis, E. coli) y a la necesidad de tratar empíricamente microrganismos anaerobios con metronidazol por su excelente actividad frente a $B$. fragilis, Prevotella y Fusobacterium, así como por las características farmacocinéticas de ambos antibióticos, que alcanzan elevadas concentraciones en el parénquima cerebral y en LCR (14). Sin embargo, en los pacientes con AC y antecedente de TCE o neurocirugía es necesario añadir al tratamiento un antibiótico con actividad frente a $S$. aureus y Estafilococos coagulasa negativa y por tanto las recomendaciones incluyen cloxacilina o vancomicina.

De esta forma se obtuvo un grupo de pacientes que recibió tratamiento antibiótico protocolizado y se comparó con el grupo inicial en el que los enfermos recibían tratamiento antibiótico empírico de acuerdo con la prescripción individualizada del médico responsable que no se atenía a protocolo terapéutico interno de nuestro centro. Las características clínico-epidemiológicas de base de ambas series eran homogéneas, excepto por la mayor edad (porcentaje de pacientes $>40$ años) en el grupo de pacientes correspondientes al segundo periodo. El número de curaciones en el PII era significativamente mayor así como la frecuencia de recidivas era menor, resultados similares a los obtenidos por otros (15). Ante estos resultados, consideramos que la Protocolización terapéutica mejora las pautas de tratamiento empírico e incide sobre uno de los factores fácilmente modificables en la práctica clínica a la hora de lograr disminuir la morbi-mortalidad de los procesos infecciosos en general y de los AC en particular. No obstante una limitación de este estudio es la diferencia entre los antibióticos empleados en el grupo II (cefotaxima y metronidazol o ceftazidima o meropenem más cloxacilina o vancomicina), que son mas activos y de más amplio espectro que los del grupo I. Sin embargo consideramos que la existencia de protocolos que faciliten la elección empírica de la pauta antibiótica es crucial ya que debe tener en cuenta las características del paciente, sus antecedentes epidemiológicos, la gravedad clínica inicial y no olvidar los cambios epidemiológicos en cada área y hospital, siguiendo las bases microbiológicas que permiten optimizar el uso racional de antibióticos (16). La persistencia de una elevada mortalidad en las series de pacientes con AC hace no obstante necesario revisar algunos de los protocolos terapéuticos (17), que tengan en cuenta la aparición de los ya no tan nuevos agentes antimicrobianos, como linezolid, activo en infecciones graves por $S$. aureus resistente a meticilina $(18,19)$ o meropenem, que aúna su gran eficacia en infecciones por Enterobacterias productoras de BLEAS y Acinetobacter baumannii con una buena penetración en Sistema Nervioso Central (14,17-19).

Como conclusiones, podemos indicar que la sospecha clínica y el diagnóstico precoz y la implantación de un protocolo de tratamiento antibiótico empírico adecuado, adaptado a cada situación clínica, junto con la actuación neuroquirúrgica precoz, constituyen las bases fundamentales del tratamiento actual de mayor eficacia en los AC.

\section{Bibliografía}

1. Schiamser SE, Barman K, Norby SR. Intracranial abscesses in adults: an analysis of 54 consecutive cases. Scand J Infect Dis 1988; 20: 1-9.

2. Maticen GE, Jonson JP. Brain Abscess. Clin Infect Dis 1997; 25: 763-79.

3. Gómez J, Poza M, Martínez Pérez M, Martínez Lage J, Hernández JL, Martín Luengo F, et al. Los abscesos cerebrales en un hospital general. Análisis de 66 casos consecutivos. Med Clin (Barc) 1991; 97: 641-4

4. McCabe WR, Jackson GG. Gram negative bacteremia. Etiology and ecology. Arch Intern Med 1962; 110: 847-55

5. Winston DJ, Murphy W, Young LS, Hewitt WL. Piperacillin therapy for serious bacterial infections. Am J Med 1980; 69: 255-61

6. NCCLS Documents M2-T4. Antimicrob Newsletter 1989; 6: 3-7

7. Beam TR, Gilbert DN, Kunin CM. General Guidelines for the clinical evaluation of anti-infective drug products. Clin Infect Dis 1992; 15 (Supl. 1): 55-32

8. Blanco García A, García Vázquez E, Benito N, de Gárgolas M, Muñiz J, Gadea I, et al. Absceso cerebral. Estudio clinicomicrobiológico y análisis pronóstico de 59 casos. Rev Clin Esp 1998; 198: 413-9

9. Sichizya K, Fieggen G, Taylor A, Peter J. Brain abscesses- the Groote Schuur experience, 1993-2003. S Afr J Surg 2005; 43: 79-82.

10. Bhand A. Brain Abscess-diagnosis and management. J Coll Physicians Surg Pak 2004; 14: 407-10.

11. Mwang'Ombe NJM. Brian abscess at the Kenyatta National Hospital, Nairobi. East Afr Med J 2000; 77: 323-5.

12. Tattevin P, Bruneel F, Clair B, Lellouche F, de Broucker T, Chevret S, et al. Bacterial brain abscesses: a retrospective study of 94 patients admitted to an Intensive Care Unit (1980 to 1999). Am J Med 2003; 115: 143-6.
13. Carpenter J, Stapleton S, Holliman R. Retrospective analysis of 49 cases of brain abscess and review of the literature. Eur J Clin Microbiol Infect Dis 2007; 26: 1-11.

14. Tunkel AR. Brain Abscess. In ( Mandell GL, Bennett JE, Dolin R. Eds.) Principles and Practice of Infectious Diseases. Philadelphia. Elsevier, Churchill-Livingstone. Sixth Edition 2005; 1150-63.

15. Jansson AK, Enblad P, Sjolin J. Efficacy and safety of cefotaxime in combination with metronidazole for empirical treatment of brain abscess in clinical practice. A retrospective study of 66 consecutive cases. Eur J Clin Microbiol Infect Dis 2004; 23: 7-14.

16. Gómez J, Baños V. Bases para optimizar el uso racional de los antibióticos en la clínica práctica. En (Gómez J y Gobernado M Eds) Enfoque Clínico de los Grandes Síndrome Infecciosos. Madrid. Editorial Ergon. Segunda Edición 2006: 331-42.

17. García Vázquez E, Gómez J. Infecciones del Sistema Nervioso Central. Meningitis y absceso cerebral. En (Serrano-Heranz R, Barberan J. Eds). Protocolos Enfermedades Infecciosas. Sociedad Española de Medicina Interna. Madrid. IMC. $2^{\mathrm{a}}$ Edición 2006; 13-37.

18. Llinares P, Sousa MD, Sánchez E. Tratamiento de las infecciones del sistema nervioso central. En (García Rodríguez JA, García Sánchez JE, Gobernado M, Picazo JJ y Prieto J Eds.) Antimicrobianos en Medicina. Barcelona. Sociedad Española de Quimioterapia. Prous Science. $2^{\mathrm{a}}$ edición 2006;495-505

19. Gómez J, García Vázquez E, Baños R, Canteras M, Ruiz J, Baños V, et al. Predictors of mortality in patients with methicillin-resistant Staphylococcus aureus (MRSA) bacteraemia: the role of empiric antibiotic therapy. Eur J Clin Microbiol Infect Dis 2007; 26: 239-45. 